Vol. 4. No. 2. (75-94). Julio-Dic/2019

ISSN: 2539-1518

\title{
Fundamentación teórica sobre lectura de los docentes de lengua castellana en las escuelas públicas de Barranquilla
}

\author{
Álvaro García Burgos \\ alvaroramon.garciaburgos@gmail.com \\ Universidad del Atlántico
}

Diego García Burgos

dagb571@hotmail.com

Universidad del Atlántico

\section{Resumen}

El objetivo de esta investigación fue determinar los factores que inciden en los procesos de formación lectora en las escuelas oficiales de estrato 1, 2, y 3 de Barranquilla, con bajos promedios en las pruebas Saber/Icfes, en el área de lenguaje. La metodología se enmarca dentro de la investigación cualitativa, mediante la aplicación de una entrevista estructurada a los docentes de instituciones educativas analiza los aspectos relacionados con la importancia de la lectura en la organización de los contenidos, los diversos teóricos desde las cuales el docente fundamenta su concepción lectora, los tipos de lectura que utiliza, el uso o no de los textos escolares y su importancia en el aula. Se encontró que los docentes no tienen en cuenta estrategias que propicien las inferencias, la reflexión, la capacidad crítica en los procesos lectores, prefieren las actividades de promoción y de lúdica a las de formación de un lector investigador, además, los docentes relacionan los factores que sus estudiantes presentan en el aula de clases: desinterés, falta de técnicas lectoras, malos hábitos lectores, pero las estrategias que utilizan después de diagnosticar el problema, no son las más efectivas. Finalmente, queda demostrada la poca claridad teórica en procesos de lectura crítica de los docentes que están a cargo de las asignaturas de lenguaje en cada una de estas instituciones, siendo este uno de los factores que inciden en los bajos resultados de los estudiantes.

\section{Palabras clave}

Docentes de escuelas oficiales, bajos promedios en pruebas Saber, teóricos de lectura, formación lectora.

Recibido 26/11/2019 - Aprobado 5/12/2019. 


\title{
Theoretical foundation on reading of teachers of the Spanish language in the public schools of Barranquilla
}

\begin{abstract}
The objective of this research was to determine the factors that affect the processes of reading training in the official schools of stratum 1, 2, and 3 of Barranquilla, with low averages in the Saber / Icfes tests, in the area of language. The methodology is part of the qualitative research, through the application of a structured interview to teachers of educational institutions the aspects related to the importance of reading in the organization of content, were analyzed through various theorists from which the teacher bases his reading conception, the types of reading they use, the use or not of school texts and their importance in the classroom. It was found that teachers did not take into account strategies that favor inferences, reflection, critical capacity in reading processes, prefer promotional and recreational activities to those of training a research reader, in addition, teachers relate the factors that their students present in the classroom: disinterest, lack of reading techniques, bad reading habits, but the strategies they use after diagnosing the problem are not the most effective. Finally, the lack of theoretical clarity in processes of critical reading of the teachers who are in charge of the language subjects in each of these institutions is demonstrated, this has being one of the factors that affect the low results of the students.
\end{abstract}

\section{Keywords}

Teachers of official schools, low averages in Saber tests, reading theorists, reading training. 


\section{Fundamentos teóricos da leitura de professores da língua espanhola nas escolas públicas de Barranquilla}

\section{Resumo}

O objetivo desta pesquisa foi determinar os fatores que afetam os processos de treinamento em leitura nas escolas oficiais dos estratos 1, 2 e 3 de Barranquilla, com baixas médias nos testes Sabre / Icfes, na área da linguagem. A metodologia faz parte da pesquisa qualitativa, através da aplicação de uma entrevista estruturada aos professores das instituições de ensino, os aspectos relacionados à importância da leitura na organização do conteúdo, foram analisados por diversos teóricos a partir dos quais o professor baseia sua concepção de leitura, tipos de leitura que usam; uso ou não de textos escolares e sua importância na sala de aula. Verificou-se que os professores não levaram em conta estratégias que favorecem inferências, reflexões, capacidade crítica nos processos de leitura, preferem atividades promocionais e recreativas às do treinamento de um leitor pesquisador, além disso, os professores relacionam os fatores que seus alunos apresentam na sala de aula: desinteresse, falta de técnicas de leitura, maus hábitos de leitura, mas as estratégias utilizadas após o diagnóstico do problema não são as mais eficazes. Por fim, é demonstrada a falta de clareza teórica nos processos de leitura crítica dos professores responsáveis pelas disciplinas de línguas de cada uma dessas instituições, sendo este um dos fatores que afetam os baixos resultados dos alunos.

\section{Palavras-chave}

Professores de escolas oficiais, baixas médias nos testes do Sabre, teóricos da leitura, treinamento em leitura. 


\section{Introducción}

Los bajos promedios en los resultados de pruebas Saber/ Icfes en la región Caribe colombiana son evidentes. Para el presente artículo, se tomaron los datos de Barranquilla (Atlántico), como representación de la región, por obtener, entre las capitales de la Costa, el resultado más cercano (visible) al promedio nacional.

Las investigaciones realizadas evidencian problemas en la enseñanza de la lectura en los departamentos de la región Caribe. Sólo el 23\% de los estudiantes de educación básica de Barranquilla/Soledad logra comprender lo que leen. El 10\% ni siquiera capta la información literal contenida en los textos (MEN - SABER, 1997 - 1999). En los resultados de la Evaluación Censal de la Calidad de la Educación en Matemáticas y Lenguaje (marzo de 2002), el $33 \%$ de los niños de $5^{\circ}$ grado no tiene el nivel mínimo de conocimientos de lenguaje, les cuesta trabajo entender lo que leen y extraer las ideas principales y menos del $10 \%$ obtuvo los logros esperados para su edad (Iriarte, 2010).

De acuerdo con el estudio de García (2017)

En la región Caribe colombiana, en el 2012, en el área de Lenguaje, el $35 \%$ de los estudiantes no alcanzó el nivel mínimo de la prueba, mientras que para el consolidado nacional este porcentaje fue de un $23 \%$. En 2014, los resultados de los grados 3, 5 y 9 se incrementaron en $20 \%$.

En el mismo estudio, se afirma que para el caso de la prueba SABER $11^{\circ}$, en un espacio de diez años, entre 2007 y 2016, los resultados muestran que en Colombia los estudiantes de educación media se ubican en el nivel mínimo con un promedio de 48,08\% en el área de lenguaje. En el año 2007, el promedio fue de 47,01\%; en 2008, de 45,44\%; en 2009, de 46,14\%; en 2010, de 46,18\%; en 2011, de 46,03\%; en 2012, de 46,5\%; en 2013, de 47,3\%; en 2014 , de 50,16\%; en 2015, de $49.7 \%$ y en 2016, de 52.6\%. Estos resultados llevan a indagar por las razones que inciden en tan bajos promedios, por lo cual se han formulado los siguientes interrogantes: ¿De qué manera la fundamentación teórica sobre lectura de los docentes afecta sus procesos metodológicos y, finalmente, los resultados de sus estudiantes? ¿Qué contenidos seleccionan para la enseñanza de la asignatura y cómo los utilizan? ¿Qué 
tipo de textos emplean para las prácticas de lectura? Diagnosticar y analizar estos factores es el propósito de esta investigación.

Para la realización se consideró pertinente partir de la revisión teórica de los conceptos de lector y de lectura, desde la perspectiva sociocultural, con el fin de contrastarlos con los manejados por los docentes de lenguaje, y en los cuales se apoyan para el desarrollo de sus propuestas metodológicas. En este sentido, sobre el concepto de lector, es pertinente retomar los aportes que hace W. Iser (1976), quien denomina investigador al lector, pues no recibe pasivamente los contenidos. El texto, según dicho autor, inicia la transferencia, la cual se logra sólo con los actos de consciencia del lector.

Esta redefinición implica una labor interesante en el proceso de lectura que se realiza a diario, pues involucra actividades de búsqueda, pasando de un texto a otro, en la persecución de un nuevo o mejor significado, en la deconstrucción de un símbolo. Se ingresa en un proceso de intertextualidad que, a la manera de Gennette (1989), conlleva un salto hacia atrás (mirar textos anteriores), hacia los lados (textos de otros géneros con la misma temática) o hacia adelante (textos elaborados a partir del texto inicial); la búsqueda por la plenitud del significado es esencial para la comprensión, valoración y trascendencia del contenido del texto leído.

Asimismo, Cassany (2009) define la lectura como una práctica letrada, inserta en prácticas sociales. Utiliza el término "práctica” para enfatizar que la lectura tiene un carácter social y se integra en todas las actividades sociales que los seres humanos realizan, tales como: informarse, trabajar, hacer trámites legales, vivir en sociedad. En ese orden de ideas, desde la perspectiva sociocultural, es importante considerar también el concepto de literacidad. Según Zavala (2008), implica una manera de usar la lectura y la escritura en el marco de un propósito social especifico. Los usos de la lectura y la escritura van más allá de la escuela, dado que leer y escribir no son fines en sí mismos: no se lee o se escribe para leer y escribir. Al contrario, son formas de lograr objetivos sociales y prácticas culturales más amplios.

En los procesos de enseñanza de la lengua, se deben aplicar métodos críticos de lectura que permitan sensibilizar a los jóvenes sobre el poder del lenguaje (Cassany, 2003, 
p.113); sobre sus usos, de acuerdo con el contexto; sobre sus interpretaciones, más allá de lo expresado; sobre la intención del enunciador y sobre su postura ideológica y epistemológica.

Esta búsqueda y construcción de significado, como lo plantea Santiago (2010), requiere que el lector efectúe una serie de operaciones cognitivas (predicción, abstracción, análisis, síntesis, inferencia, comparación) en las que pone en juego conocimientos, intereses y estrategias con los aspectos que proporciona el texto como tal, en unas circunstancias determinadas. De esta forma, la lectura se torna en una interacción entre lector, texto y contexto. Este proceso, sin duda, se constituye en uno de los caminos para llegar a la construcción de un lector crítico, capaz de interpretar un texto desde distintas dimensiones.

Es pertinente señalar que los Estándares Básicos de Lenguaje propuestos por el Ministerio de Educación Nacional de Colombia para el ciclo de $6^{\circ}$ a $9^{\circ}$ coinciden con los presupuestos anteriormente expuestos.

Así presenta el MEN estas competencias:

Comprendo e interpreto diversos tipos de textos, para establecer sus relaciones internas y su clasificación en una tipología textual. Para lo cual, reconozco las características de los diversos tipos de texto que leo. Propongo hipótesis de interpretación para cada uno de los tipos de texto que he leído. Identifico las principales características formales del texto: formato de presentación, títulos, graficación, capítulos, organización, etc. Comparo el contenido de los diferentes tipos de texto que he leído. Relaciono la forma y el contenido de los textos que leo y muestro cómo se influyen mutuamente y establezco relaciones de semejanza y diferencia entre los diversos tipos de texto que he leído (pp. 36-37).

Este modelo de lector y esta concepción sobre el proceso de lectura se toman como referentes para la elaboración y el análisis del instrumento aplicado en esta investigación, con el propósito de llevar a cabo la recolección de la información.

Se espera que los resultados que arroje la aplicación de dicho instrumento, estructurado a partir de cinco tópicos, permitan determinar los factores que pueden incidir en 
el desarrollo lector de los estudiantes de las escuelas oficiales de los estratos 1, 2 y 3 de Barranquilla, con bajos promedios en las pruebas Saber/Icfes en el área de lenguaje.

\section{Metodología}

En el curso de esta investigación cualitativa, sobre los factores que pueden incidir en el desempeño lector de los estudiantes, se decidió entrevistar a 100 docentes del área de lenguaje de 27 escuelas públicas del distrito de Barranquilla que obtuvieron bajo rendimiento según las pruebas Saber/Icfes. Las instituciones seleccionadas se encuentran ubicadas en los estratos 1, 2 y 3, sectores económicamente desfavorecidos en el distrito de Barranquilla, para indagar sobre la importancia de la lectura en la organización de los contenidos, las posturas teóricas desde las cuales los docentes fundamentan su concepción lectora, los diversos tipos de textos que utilizan para favorecer el desarrollo lector en sus estudiantes, la utilización o no de libros (textos-guía) escolares; además se examinó sobre la inclusión y pertinencia de contenidos socioculturales en los procesos de lectura por parte de los profesores en el aula de clase. El estudio se realizó entre julio y septiembre de 2010, y sus conclusiones se han visto ratificadas por investigaciones posteriores sobre la misma problemática, como ya se enunció anteriormente. Se convino confidencialidad sobre el nombre del docente y de la institución.

A la muestra, se le realizó una entrevista estructurada escrita, basada en cuarenta (40) preguntas, de las cuales el $25 \%$ era de carácter cerrado, pues interesaba conocer el porcentaje y la frecuencia de algunas prácticas en los docentes de lengua castellana del distrito de Barranquilla; y el $75 \%$ de carácter abierto, ya que para este análisis era fundamental identificar los presupuestos teóricos, metodológicos y epistémicos sobre el proceso de lectura manejados por dichos docentes. La entrevista fue validada por pares expertos en Lingüística y en Didáctica de la Universidad del Atlántico, y se estructuró a partir de cinco tópicos básicos, que se describen a continuación:

Tipología textual: las preguntas de este tópico pretenden evidenciar la diversidad de textos que utiliza el docente, las actividades que realiza y los contenidos específicos que desarrolla en el aula de clase. 
Motivación hacia la Lectura: las preguntas de este tópico intentan indagar si el docente de lengua tiene en cuenta, para la selección de los contenidos, los factores que influyen en el proceso de lectura.

Referentes teóricos sobre lectura: las preguntas elaboradas sobre este tópico pretenden identificar los fundamentos teóricos desde los cuales el docente de lenguaje orienta el proceso lector con sus estudiantes, como también los niveles de lectura que privilegia en el aula de clase.

Libros escolares: las preguntas diseñadas sobre este tópico tratan de establecer la importancia que el docente de lenguaje le da al libro escolar (texto-guía) para la enseñanza de la lectura, cuántas unidades del texto utiliza, si recurre a otros materiales de lectura o si sólo privilegia los del texto escolar.

Identidad: las preguntas de este tópico pretenden evidenciar la inclusión de contenidos socioculturales, como, por ejemplo, la diversidad cultural de la región Caribe, por parte de los docentes de lenguaje, en las prácticas de lectura que adelantan con sus estudiantes en el aula de clase.

\section{Resultados}

Valoradas las respuestas de los 100 docentes de las 27 escuelas públicas del distrito de Barranquilla, que corresponden a la muestra de esta investigación, se encontró lo siguiente:

Cuando se les preguntó sobre el porcentaje de importancia que le daban a la lectura en la organización de los contenidos, el $40 \%$ de los docentes respondió que el 100\%; el 20\% manifestó que el 90 \%; el 10\% expresó que el $80 \%$ y el 30\%, el 70\%. Es decir, estos datos demuestran que la lectura tiene un alto porcentaje de importancia al momento de organizar la clase de lenguaje en los maestros entrevistados. 
Al indagar por el uso de los diversos tipos de textos que privilegian los docentes de las escuelas públicas seleccionadas para propiciar el desarrollo del proceso lector en sus estudiantes, se encontró que los entrevistados reconocen la importancia de incluir todos los tipos de textos, cuando definen los contenidos en su programación anual, y manifiestan que los utilizan en el aula de clases.

Sin embargo, al precisar la frecuencia de uso de los diversos tipos de textos, se puede observar, en el diagrama (1), que el texto narrativo es el que más se emplea, es decir, los docentes privilegian actividades que giren en torno a narrar, tanto de manera oral como escrita.

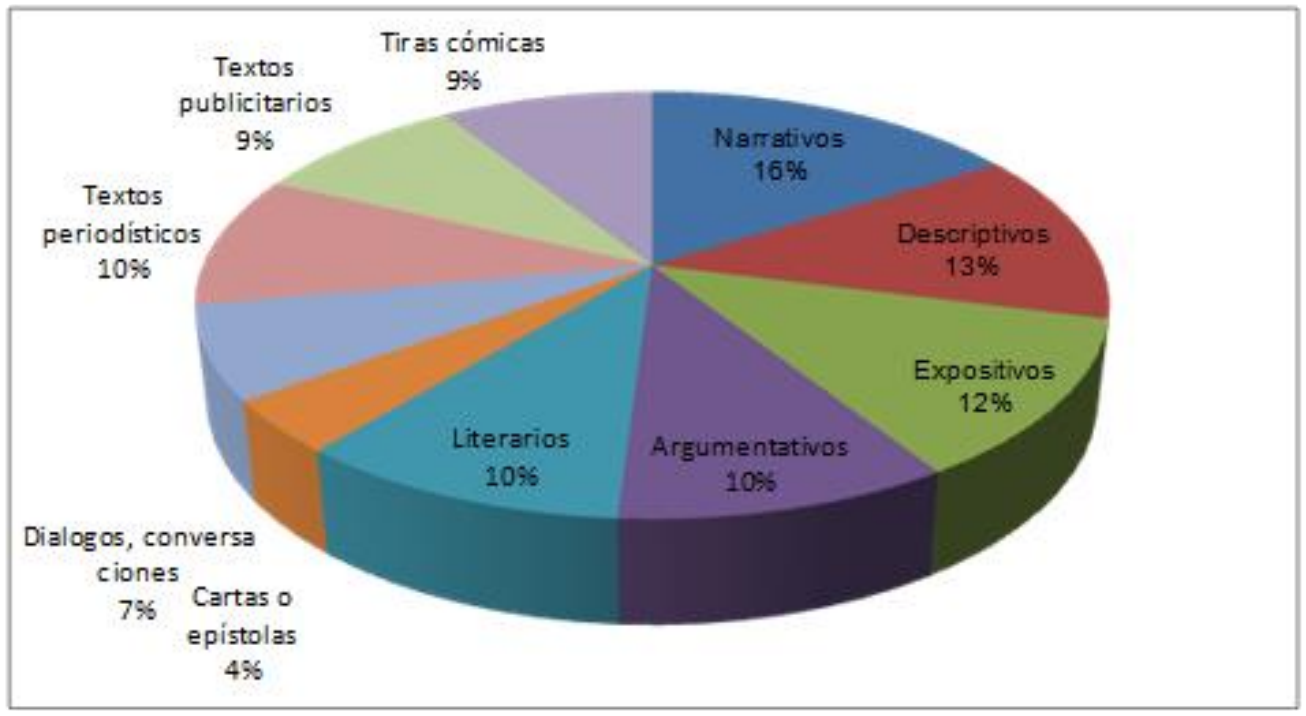

Diagrama 1. Tipos de textos

En cuanto a las actividades que se realizan alrededor de una lectura, se constató que los docentes privilegian las de expresión oral (66\%), sobre las de producción escrita (34\%). (Tabla 1). Es pertinente señalar, al observar estos datos, que los procesos de inferencia, el leer entre líneas, la capacidad de recuperar los implícitos subyacentes en el texto, que contribuyen de manera decisiva a elaborar su coherencia global, a construir el significado 
relevante del escrito y a dilucidar puntos de vista diferentes (Cassany, 2003) ocupen menos la atención del docente que la socialización de lectura, que se reduce sólo a la comprobación de si el estudiante leyó o no el texto seleccionado.

Tabla 1

Actividades alrededor de la lectura

\begin{tabular}{|c|c|c|c|c|c|}
\hline $\begin{array}{l}\text { Actividades } \\
\text { escritas }\end{array}$ & Porcentaje & $\begin{array}{l}\text { Porcentaje } \\
\text { total }\end{array}$ & $\begin{array}{l}\text { Actividades de } \\
\text { expresión oral }\end{array}$ & Porcentaje & $\begin{array}{l}\text { Porcentaje } \\
\text { total }\end{array}$ \\
\hline $\begin{array}{ll}- & \text { Carteleras } \\
- & \text { Ensayos } \\
- & \text { Reseñas } \\
- & \text { Periódicos } \\
- & \text { Crucigramas } \\
- & \text { Sopas de } \\
& \text { letras } \\
- & \text { Creación de } \\
& \text { textos } \\
\text { - Diapositivas }\end{array}$ & $\begin{array}{c}10.5 \% \\
2.7 \% \\
3 \% \\
2 \% \\
5 \% \\
5 \% \\
5.3 \% \\
0.5 \%\end{array}$ & $34 \%$ & $\begin{array}{ll}- & \text { Foros } \\
- & \text { Socialización de la } \\
& \text { lectura } \\
- & \text { Mesa redonda } \\
- & \text { Debate } \\
- & \text { Exposiciones } \\
- & \text { Videos } \\
- & \text { Dramatizaciones }\end{array}$ & $\begin{array}{l}5.7 \% \\
15 \% \\
\\
9.3 \% \\
5.7 \% \\
15 \% \\
0.3 \% \\
15 \%\end{array}$ & $66 \%$ \\
\hline
\end{tabular}

Cabe anotar que, en el grupo de docentes entrevistados, las actividades de carácter escrito después de la lectura tienen una frecuencia del 100\%. Sin embargo, estas actividades tienen que ver con la construcción de carteleras (10,5\%), crucigramas $(5,0 \%)$, sopa de letras $(5,0 \%)$ para un total de $20,5 \%$, mientras que las actividades de creación de textos $(5,3 \%)$ y la construcción de reseñas $(3,0 \%)$ y de ensayos $(2,7 \%)$ sobre el texto leído corresponden al 11,0\%. Es evidente que los docentes, en planeación y organización de las actividades, no tienen en cuenta estrategias que propicien la inferencia, la reflexión, la capacidad crítica en el proceso lector, pues prefieren las actividades de promoción y de lúdica a las de formación de un lector investigador, tal como lo plantea Iser (1987).

Lo que concierne a los factores que inciden en la motivación hacia la lectura, en la entrevista, se consultó a los docentes sobre los aspectos que pueden afectar negativamente en el proceso de formación lectora de los estudiantes. Los resultados fueron los siguientes: diagrama (2). 


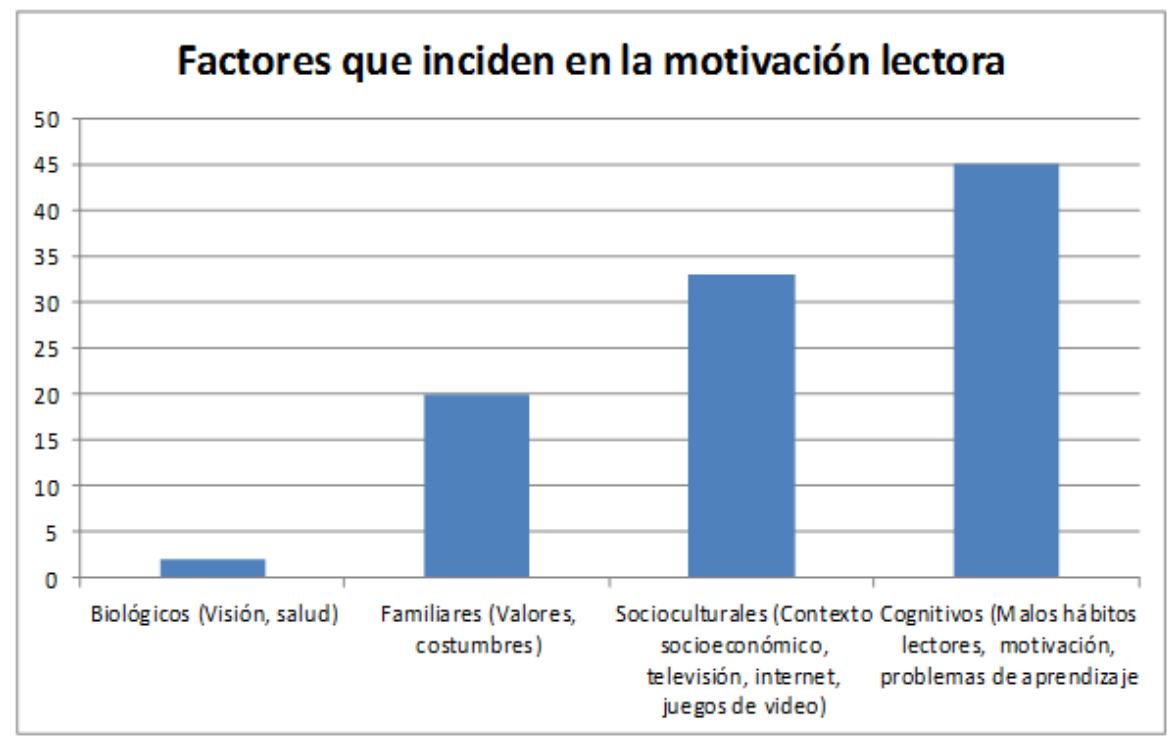

Diagrama 2. Factores que inciden en la motivación lectora

Los maestros consideraron que el factor que más incide en el desarrollo lector, es el que tiene que ver con problemas cognitivos de los estudiantes (45\%), de los cuales destacaron las dificultades de aprendizaje, los malos hábitos lectores, falta distracción, entre otros. Con respecto al aspecto sociocultural, como elemento que afecta el proceso lector, el 33\% respondió que el contexto socioeconómico en que les toca vivir a los estudiantes, alrededor de su escuela, mayormente de estrato 1 y 2 , incide de manera fundamental en la formación y los intereses del joven, como también la influencia de la televisión, los juegos de video y el internet.

En cuanto al aspecto familiar, como se describe en el diagrama 2, el $20 \%$ manifestó que influye el poco acceso a los libros, la falta de costumbre lectora de los padres y hermanos, y el bajo nivel de escolaridad de los padres.

Por último, el 2\% respondió que los problemas de visión, o las deficiencias en salud en general, contribuyen al desinterés de los estudiantes por la lectura y, por tanto, afectan el proceso lector.

Al interpretar los resultados sobre la motivación hacia la lectura, se observa que los docentes atribuyen como causas de la baja motivación que presentan al respecto los 
estudiantes, las siguientes: desinterés, falta de técnicas lectoras, malos hábitos lectores. Pero los bajos resultados demuestran que las estrategias que utilizan, después de diagnosticar el problema, no son las más efectivas. Por otro lado, se desconoce que el interés por la televisión y el internet por parte de los jóvenes, es un factor que puede contribuir, manejado metodológicamente en forma adecuada, a fortalecer el proceso de lectura crítica.

Otro de los tópicos acerca de los cuales se les indagó a los docentes de estos centros educativos, fue la utilización de teorías o teóricos sobre la lectura, desde las cuales fundamentan su práctica pedagógica sobre el proceso lector. El 74\% respondió afirmativamente sobre la utilización de una teoría como guía y el 36\% reconoció no utilizar ninguna. (Diagrama 3).

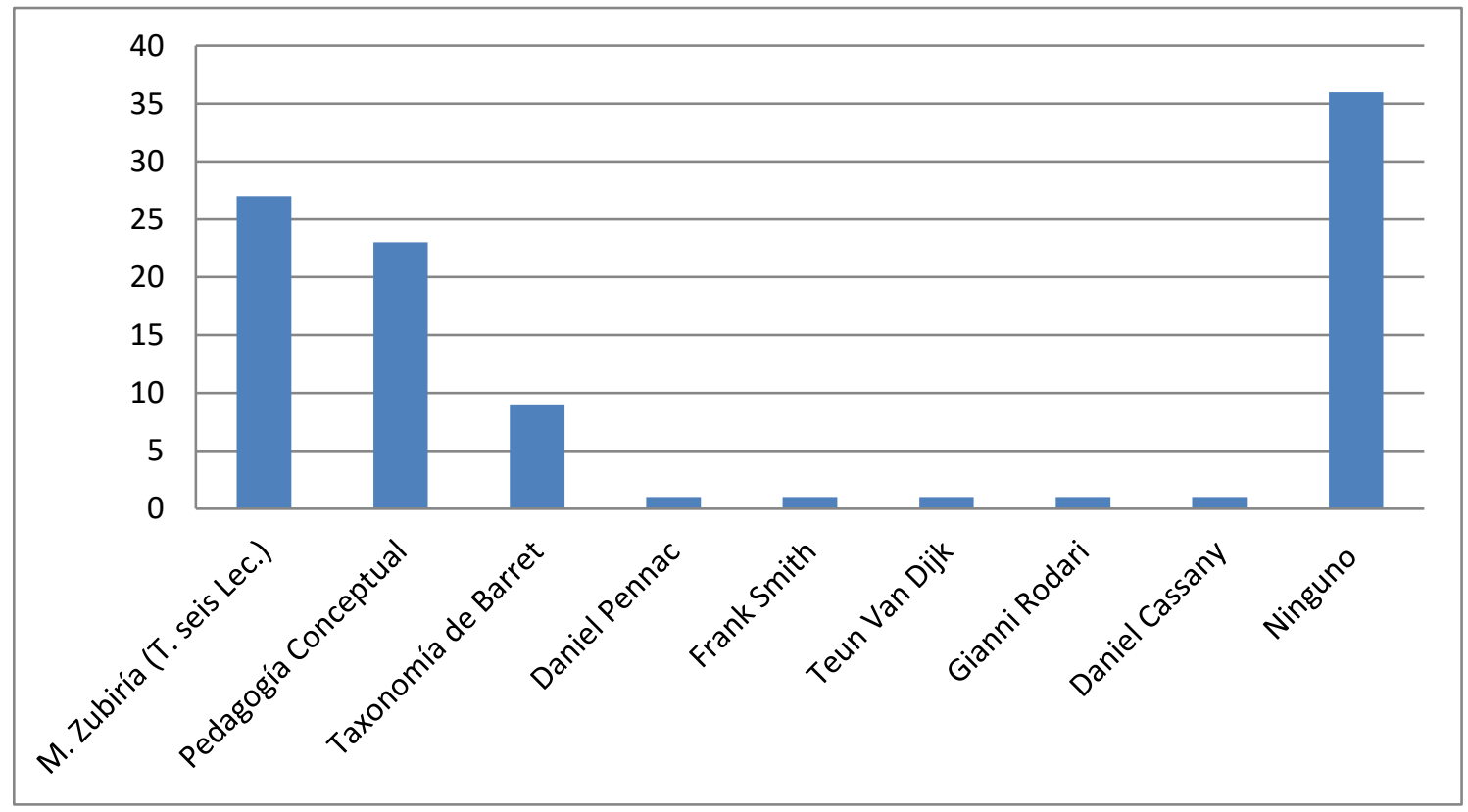

Diagrama 3. Teóricos que fundamentan el proceso lector

$\mathrm{Al}$ analizar las respuestas de quienes contestaron afirmativamente, observamos que el 27\% reconoció utilizar la "teoría de las seis lecturas" de Miguel de Zubiría; el 1\%, a Frank Smith, Teun Van Dijk y Daniel Cassany. Es decir, el 30\% reconoce teóricos de lectura, que 
es un porcentaje menor, con respecto al resto de maestros entrevistados; además, valdría la pena analizar cómo los utilizan, qué tiempo tienen de aplicar sus planteamientos en la escuela donde laboran, cómo inciden en las políticas de lectura de su institución o si son prácticas aisladas, pues sus resultados no son evidentes todavía. (Esto sería digno de otra investigación).

El 23\% usa la Pedagogía Conceptual. Sobre el particular, ex pertinente aclarar que éste es un modelo pedagógico orientado al desarrollo de la inteligencia en todas sus manifestaciones; por tanto, su correcta aplicación, con una excelente guía de lectura, podría llevar a los estudiantes a lograr un gran nivel de formación en todos los campos, pero no es una teoría de lectura.

El 1\% se basa en el decálogo planteado por Daniel Penacc, el cual contagia el amor por la lectura, pues la concibe como un placer, y es esencial que el estudiante así la perciba. Sin embargo, con el fin de formar un lector crítico, los aportes de Penacc se deben complementar con propuestas metodológicas que inciten al estudiante a indagar en otros textos, a desentrañar los símbolos implícitos y, en otras palabras, como plantea Cassany (2003:116), citando los planteamientos de Gray (1960), leer "las líneas", leer "entre líneas" y leer "detrás de las líneas".

El mismo sentido del placer por la lectura y la literatura infantil y juvenil, es planteado por Gianni Rodari, y todos los docentes deberían leer la Gramática de la fantasía (1999), para propiciar un espacio de re-creación del texto leído y de lúdica en sus clases; pero el reto de la formación del lector competente o modelo, como plantea Eco (1987), no alcanza a desarrollarse en dicha perspectiva.

Por otra parte, el 34\% de los docentes respondió negativamente con respecto a la utilización de algún teórico para fundamentar el proceso lector en las aulas de clases; este porcentaje, más el 23\% que asumió la Pedagogía Conceptual, el 2\% que se basa en Penacc y Rodari, nos arroja el 59\%. Este resultado permite inferir que un significativo número de docentes de escuelas públicas del distrito de Barranquilla, con bajos resultados en las pruebas Saber/Icfes, no tiene claridad conceptual sobre los teóricos que fundamentan el proceso de lectura, factor que incide en las estrategias metodológicas que utilizan y, consecuentemente, en el desarrollo lector alcanzado por sus estudiantes. 
Otro de los tópicos investigados fue el texto escolar (texto-guía), por ser empleado en muchas instituciones en el área de lenguaje y ser considerado como herramienta clave en el aprendizaje y la enseñanza de la lengua. La entrevista incluyó preguntas como: si usaban o no textos escolares, cuántas unidades del texto-guía manejaban y qué tipo de textos escolares solían emplear en sus clases. (Diagrama 4).

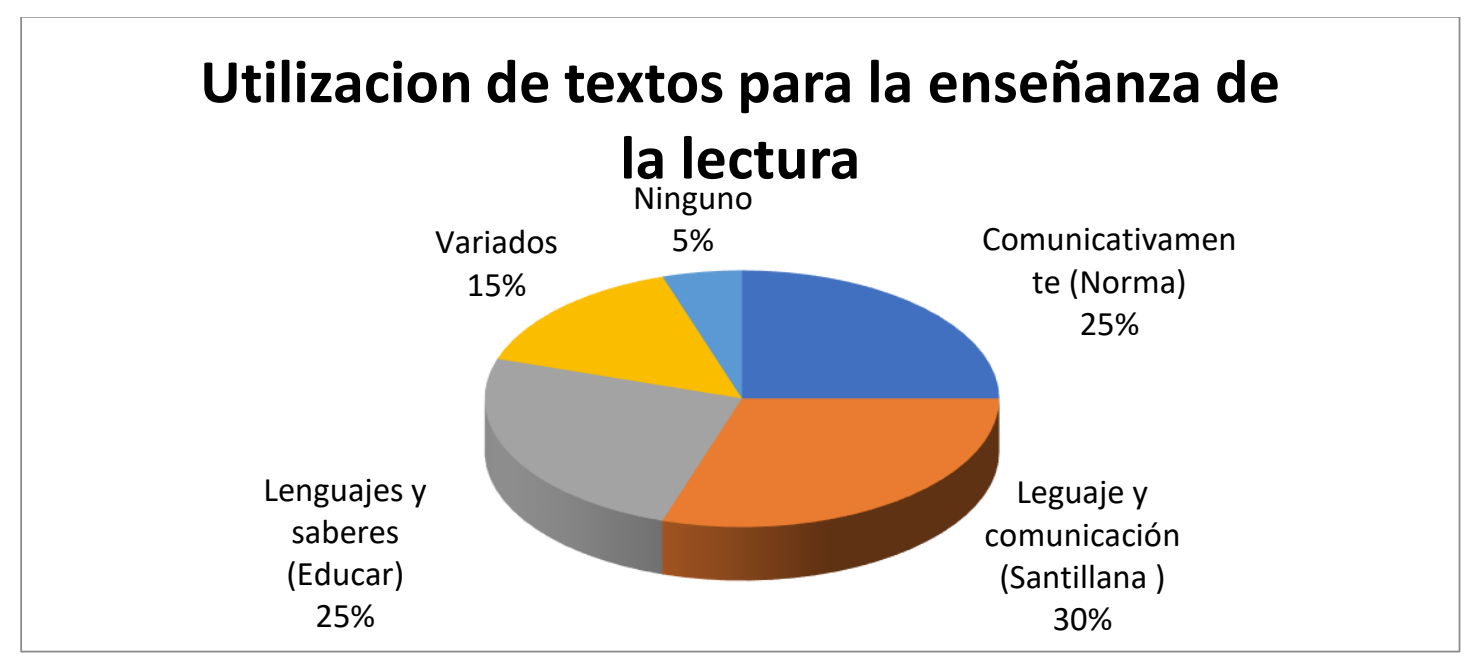

Diagrama 4. Utilización de textos para la enseñanza de la lectura

Como se puede constatar en el diagrama 5, el 95\% de los docentes utiliza los textos escolares de lenguaje. Con respecto a los tipos de texto escolar que privilegia el maestro para sus clases, los resultados expresan que el $30 \%$ prefiere los talleres de lectura; el $20 \%$, libros sobre temas gramaticales; el 20\%, textos literarios; el 15\%, lingüísticos y el 15\%, talleres de escritura.

Los docentes reconocen al texto escolar como un buen apoyo para la realización de sus clases, y los porcentajes del diagrama 5 demuestran la importancia que le dan para el desarrollo del proceso lector en sus estudiantes. Es así que, algunos docentes de las escuelas públicas con promedios bajos por parte de sus estudiantes en la prueba Saber/Icfes de lenguaje, consideran que uno de los factores que inciden en los resultados tiene que ver con la no adquisición de dichos textos por todos los estudiantes, debido a sus costos. Sobre el particular, es pertinente contemplar el hecho, al realizar el análisis de los datos que arrojan 
las pruebas Saber/Icfes, que los mismos textos escolares son utilizados en otras instituciones públicas y privadas con resultados exitosos por parte de sus estudiantes en dichas pruebas. De lo anterior, se puede colegir que existen aspectos que van más allá del uso o no del texto escolar en el aula de clases y que pueden tener mayor incidencia en el desempeño lector de los estudiantes; es el caso de la insuficiente claridad teórica sobre el proceso lector que puedan tener los docentes, razón por la cual la metodología que emplean para orientar el desarrollo de la lectura en sus estudiantes no produce eficientes resultados.

Por último, en lo concerniente a la inclusión de contenidos socioculturales $y$, concretamente, la valoración de los diversos contextos de interacción de sus estudiantes, por parte del docente de lenguaje, para los ejercicios de lectura, la mayoría de los maestros consultados le dio significativa importancia; no obstante, se encontró que el $40 \%$ respondió que el texto guía sólo algunas veces contempla artículos referentes a la diversidad cultural de la región Caribe y el 60\% contestó que nunca. (Diagrama 5).

\section{Contenidos socioculturalęsen en la clase}

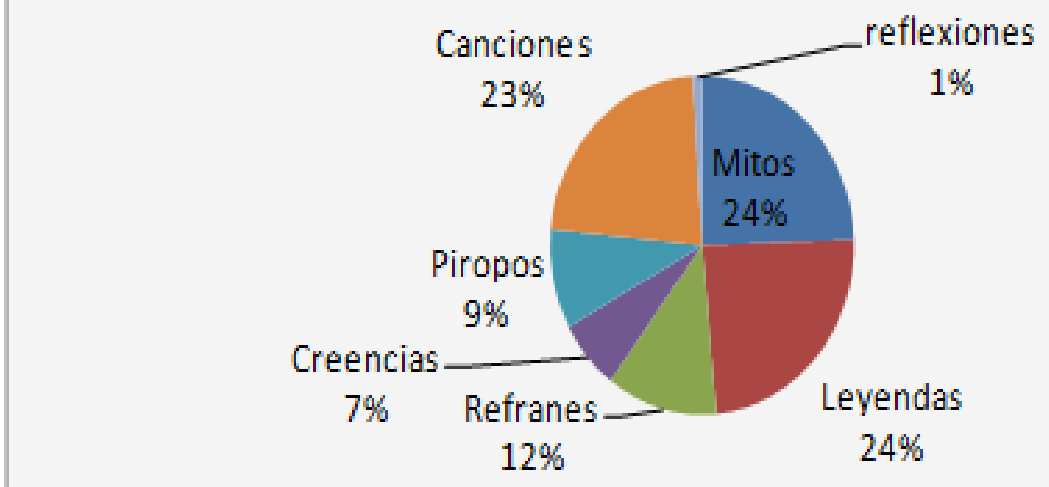

Diagrama 5. Contenidos socioculturales en la clase

En otras palabras, en la mayoría de los textos escolares, los mitos, las leyendas, los héroes de la región Caribe son poco contemplados. Al respecto, vale la pena anotar que el desconocimiento de lo vernáculo en las prácticas de lectura, podría generar una negación del legado cultural e incidir en la construcción de su identidad. 


\section{Discusión}

Con base en los resultados arrojados por la entrevista realizada, se podría deducir que los conceptos de lector y de lectura, desde los cuales parten los docentes de las escuelas públicas de Barranquilla, con bajos resultados en las pruebas nacionales, para orientar las prácticas de lectura en sus estudiantes, no corresponden a la visión de un lector investigador y no contribuyen a propiciar el desarrollo lector, en el sentido de Cassany, al no concebir la lectura como un proceso de apropiación, interpretación y trascendencia de significados: "leer las líneas", “leer entre líneas" y “leer detrás de las líneas”. Esto es, el desempeño en lectura alcanzado por los estudiantes deja entrever que, los docentes, a pesar de que utilizan variadas estrategias metodológicas en sus clases, estas actividades se centran más en la animación a la lectura que en la formación de un lector crítico.

En otras palabras, a partir de esta investigación, se podría concluir que si los docentes que están a cargo de la asignatura de lenguaje en cada una de las escuelas públicas de los estratos uno, dos y tres de Barranquilla seleccionadas no tienen claridad teórica sobre el proceso de lectura crítica, difícilmente podrían favorecer el desarrollo de dicho proceso en sus estudiantes y ésta sería una posible explicación de los bajos resultados que éstos tuvieron.

Es claro que la formación crítica de los estudiantes no es responsabilidad exclusiva de los docentes de español, sino que es responsabilidad de todo el cuerpo docente. Si los educadores no propician el diálogo de saberes entre las distintas áreas del conocimiento, difícilmente se podrá formar lectores críticos. Este sería un punto de partida para el análisis sobre las deficiencias del lector hoy en día y sobre la responsabilidad social que tiene la universidad en la formación de las nuevas generaciones de educadores.

Esto plantea un problema sobre cómo debe ser la formación de las nuevas generaciones, es decir, su orientación desde una perspectiva disciplinar, interdisciplinar y transdisciplinar, con miras a que estructuren una visión holística. Ése sería el camino para la formación de un lector investigador, de un ciudadano crítico: un estudiante que sea capaz de establecer relaciones entre el texto que lee y su hipertexto, de identificar la tesis planteada por el autor y los argumentos en los cuales se apoya y contrastarlos con otros textos sobre el mismo tema de diferentes perspectivas teóricas, es decir, de articular el conocimiento que se 
encuentra en ese discurso con las diferentes ciencias que pueden, en un momento dado, tener referencia con esa temática.

De acuerdo con los resultados analizados, se concluye que hay prácticas pedagógicas que no indagan por la realidad, que no son efectivas en la construcción de un lector crítico. Este hecho, aunado a la poca claridad teórica por parte de los docentes del área de lenguaje sobre el proceso de lectura crítica, puede incidir en el desarrollo lector alcanzado por los estudiantes.

En ese orden de ideas, los docentes de las escuelas públicas analizadas, aunque realizan actividades de promoción de la lectura en clase, éstas no se orientan a la formación de un lector crítico. Es así que, como plantea Lomas (1999), entre el deseo y la realidad, entre esta voluntad de contribuir al desarrollo de la competencia comunicativa del alumnado y el modo en que a veces se seleccionan los contenidos lingüísticos y literarios y se organizan las tareas del aprendizaje en las aulas, a menudo se abre un abismo.

Investigaciones realizadas al respecto, Iriarte (2010), Duran, Jaraba y Garrido (2007), Pimiento, M. (2012), no identifican la poca formación teórica de los docentes sobre el proceso lector como un factor que incida en los resultados deficientes de los estudiantes en lectura.

En tal sentido, esta investigación aporta información valiosa, y no contemplada antes, sobre una de las posibles causas del bajo desempeño en lectura de los estudiantes, como es el hecho de considerar la escasa fundamentación teórica que tienen los docentes sobre lectura crítica y, en particular, sobre cómo se forma un lector investigador. Resalta esta investigación que el no manejo de estos presupuestos teóricos por parte de los docentes, a su vez, podría dificultarles la elaboración y aplicación de propuestas metodológicas eficientes, es decir, que permitan la formación de un lector crítico, capaz de interpretar los aspectos culturales e ideológicos dl contexto en el que vive.

\section{Recomendaciones}

Para la formación de un lector crítico, es fundamental que el docente, igualmente, adquiera un formación holística, esto es, que se apropie de los conocimientos aportados por 
las áreas que estudian los distintos procesos que se dan en la escuela, tales como la antropología de la educación, la psicología de la educación, la sociología de la educación, la psicología cognitiva, la psicolingüística, etc. para poder interpretar la realidad de su aula y convertirla en objeto de investigación. De ahí que, este estudio debe ser tenido en cuenta por las facultades de educación de las universidades de nuestra región para que reorienten la formación de sus futuros licenciados tanto del área de lenguaje como de las otras áreas.

Los resultados de esta investigación, a su vez, permiten poner en conocimiento a las autoridades distritales, departamentales y nacionales en el área de educación uno de los factores específicos que podrían afectar el desempeño en lectura de los estudiantes de las escuelas públicas; de ahí que, sería importante que adelantaran programas de capacitación orientados a enriquecer la fundamentación teórica sobre lectura en los docentes. 


\section{Referencias bibliográficas}

Durán, Jaraba y Garrido (2007). Desarrollo de competencias lectoras a partir de un programa de acompañamiento en estudiantes de educación básica del municipio de Galapa/ Atlántico. Grupo de sexto grado de educación básica secundaria de escuela pública. Barranquilla. Fundación Universidad del Norte.

García, A. (2017). Concepciones de lector, lectura y comprensión lectora subyacentes en los textos escolares de lenguaje. Barranquilla. RUDECOLOMBIA, Doctorado en Ciencias de la Educación -CADE ATLÁNTICO.

Genette, G. (1989). PALIMPSESTOS. La literatura en segundo grado. Teoría y crítica literaria. Taurus. Madrid.

Icfes. Saber $5^{\circ}$ y $9^{\circ} 2009$. Resultados Nacionales. Resumen Ejecutivo. Recuperado de www.icfes.gov.co/.../6-informe-saber-5-y-9-2009-resultados-nacionales.

Icfes (2007-2014). Generador de Reportes de Datos Históricos. Recuperado de file://C:/Users/Alvaro/Downloads/H1NN111P07142C93ANCR.pdf

Icfes (2016). SABER 3 $3^{\circ}, 5^{\circ}$ y $9^{\circ}$. Resultados nacionales 2009 -2014. Recuperado de file://C:/Users/Alvaro/Downloads/Resultados\%20nacionales\%20saber\%203\%205\%209\%

Icfes (2016). Resumen ejecutivo Colombia en PISA 2015. Bogotá.

Iriarte Díaz-Granados, F. (2010). Evaluación del desempeño lector de un grupo de sexto de grado de una institución privada de provincia. Instituto de estudios superiores de educación. Eureka. Extraído el día 11 de diciembre de 2010: http://www.uninorte.edu.co/divisiones/iese/lumen/ediciones/4/index.html

Iser, W. (1987). El acto de leer. Madrid: Taurus.

Cassany, D. (Comp.). (2009). Para ser letrados. (Voces y miradas sobre la lectura). Barcelona: Paidós.

Anagrama.

(2006). Tras las líneas (Sobre la lectura contemporánea). Barcelona:

(2003). Aproximaciones a la lectura crítica: teoría, ejemplos y reflexiones.

Tarbiya. Revista de Investigación e Innovación Educativa. № $321^{\circ}$ semestre de 2003. p. 113-132. Instituto Universitario de Ciencias de la Educación, Universidad Autónoma de Madrid. 
Ministerio de Educación Nacional. (2006). Estándares Básicos de Competencias del Lenguaje. Bogotá: Ministerio de Educación Nacional. Pimiento, M. (2012) Las Concepciones de los Docentes sobre Competencias en Lectura y Escritura en la Formación de los estudiantes en áreas diferentes a Lengua Castellana. Universidad tecnológica de Pereira.

Zavala, V. (2008). La literacidad o lo que la gente hace con la lectura y la escritura. Textos de didáctica de la Lengua y la Literatura. N 47. P.71.79.

(2008). La literacidad o lo que la gente hace con la lectura y la escritura. Textos de didáctica de la Lengua y la Literatura. N 47. P.71.79

Santiago Galvis (2010) A. Estrategias de aprendizaje y enseñanza de la lectura. Universidad Pedagógica Nacional. Consultado el dia 11 de diciembre 2010 : http://www.buenastareas.com/ensayos/Estrategias-De-prendizaje-Y-Ensenanza-

De/1316277.html 